\title{
Comunicação/Communication
}

\section{Prevalência e aspectos epidemiológicos de enteroparasitoses na população geronte de Parnaíba, Estado do Piauí}

\author{
Prevalence and epidemiological aspects of intestinal parasites in elderly of Parnaíba, \\ State of Piaui
}

\author{
Luis Fernando Viana Furtado ${ }^{1}$ e Ana Carolina Fonseca Lindoso Melo ${ }^{2}$
}

\begin{abstract}
RESUMO
Introdução: Visto que as enteroparasitoses representam problemas de saúde pública, o objetivo deste trabalho foi determinar alguns aspectos epidemiológicos destas patologias em idosos de Parnaíba. Métodos: Através do método de Hoffman, analisou-se 294 amostras fecais de indivíduos com idade igual ou superior a 65 anos. Em cada participante aplicou-se um questionário utilizado para análise descritiva da população estudada. Resultados: A prevalência de enteroparasitose foi de $40,5 \%$. Os questionários revelaram resultados alarmantes no que diz respeito ao grau de insalubridade ao qual a população está inserida. Conclusões: Existe alta prevalência de enteroparasitoses em idosos de Parnaíba, indicando um estado epidemiológico preocupante.

Palavras-chaves: Enteroparasitoses. Idosos. Saúde Pública.
\end{abstract}

\begin{abstract}
Introduction: Since intestinal parasites pose public health problems, this work aimed to determine certain epidemiological aspects of these pathologies in elderly individuals from Parnaiba. Methods: Using the method of Hoffman, 294 fecal samples from individuals 65 years-old or over were analyzed. Each participant responded to a questionnaire used for description analysis of the population. Results: The prevalence of intestinal parasites was $40.5 \%$. The questionnaires showed alarming results regarding the noxious conditions in which this population is inserted. Conclusions: High prevalence of intestinal parasites exists among the elderly population of Parnaiba, indicating an epidemiological state of some concern.
\end{abstract}

Keywords: Intestinal parasite. Elderly. Public Health.

No Brasil, o aumento da expectativa de vida é uma das mudanças demográficas mais importantes dos últimos tempos. Isso se deve, sobretudo, ao desenvolvimento da ciência, a qual permite a cura de doenças que antes eram impassíveis de tratamento. $\mathrm{O}$ país passou a ter um caráter senil e o envelhecimento tornou-se um desafio para a saúde pública ${ }^{1}$.

O envelhecimento vem acompanhado de fragilidades naturais, tornando o idoso suscetível ao desenvolvimento de diversas doenças ${ }^{2}$. Nessa faixa etária, prevalecem condições crônicas e agudas, que cursam com crescente dependência ${ }^{1}$.

Quando se trata de enteroparasitoses, existem poucos trabalhos publicados abordando essas patologias em idosos ${ }^{3}$. Isto acontece

1. Curso de Biomedicina, Universidade Federal do Piauí, Parnaíba, PI. 2. Departamento de Biomedicina, Universidade Federal do Piauí, Parnaíba, PI.

Endereço para correspondência: Dra. Ana Carolina Fonseca Lindoso Melo. Dept ${ }^{\circ}$ Biomedicina/UFPI. Av. São Sebastião 2819, Campus Universitário Ministro Reis Veloso, 64202-020 Parnaíba, PI.

e-mail: Ifvfurtado@gmail.com

Recebido para publicação em 05/07/2010

Aceito em 16/09/2010 porque estudos apontam que com o avanço da idade o indivíduo tem tendência a adquirir melhores hábitos e um amadurecimento da resposta imune, o que diminui a prevalência dessas doenças ${ }^{4}$. Todavia, nem sempre é isso o que ocorre. Para $\mathrm{Wu} \mathrm{cols} \mathrm{s}^{5}$, com o envelhecimento do sistema imune, o indivíduo torna-se mais suscetível ao desenvolvimento de enteroparasitoses.

No Brasil, essas doenças ocorrem nas diversas regiões do país, seja em zona rural ou urbana e em diferentes faixas etárias ${ }^{3,4,6,7}$, constituindo um problema de saúde pública ${ }^{4,8}$. Todavia, são mais prevalentes nas populações que dispõem de deficitárias condições sanitárias e de saúde 4 , como é o caso da Cidade de Parnaíba, Piauí.

No Estado do Piauí, têm-se relatado elevadas prevalências de enteroparasitoses ${ }^{6,7,9}$. Entretanto, é importante salientar que não existe nenhum relato na literatura que revele a prevalência dessas patologias na população idosa piauiense. Isto demonstra a necessidade de maiores levantamentos epidemiológicos nesse contingente populacional.

No presente trabalho, objetivou-se determinar a prevalência de enteroparasitoses em idosos da Cidade de Parnaíba, Piauí, bem como correlacioná-la com seus possíveis fatores de risco.

Durante os meses de setembro de 2008 a setembro de 2009, foram selecionados, aleatoriamente, 294 indivíduos com idade igual ou superior a 65 anos, de ambos os sexos, residentes na zona urbana da Cidade de Parnaíba. Visitou-se as residências dos idosos e explicou-se sobre a realização da pesquisa. As famílias que declararam que os idosos não possuíam condições de participar do estudo, seja por debilidade física ou mental, não foram incluídas no trabalho. A condição financeira dos idosos não foi pré-requisito para inclusão dos mesmos na pesquisa; portanto, participaram do estudo idosos de diferentes classes sociais.

As fezes foram coletadas individualmente e acondicionadas em potes plásticos com tampa, devidamente identificados. Este material foi fixado com MIF (mercúrio, iodo e formol) e analisado através do método de sedimentação espontânea.

Foi aplicado em cada participante um questionário padrão, baseado em um conjunto de questões objetivas e de múltipla escolha, cujos resultados foram utilizados para análise descritiva da amostra estudada.

O trabalho foi aprovado pelo Comitê de Ética da Universidade Federal do Piauí e cada participante assinou um termo de consentimento autorizando a utilização do material coletado para fins de pesquisa. Todos os voluntários receberam o resultado do exame coproparasitológico. Os idosos positivos para alguma enteroparasitose foram orientados a se encaminhar às unidades de saúde do município para tratamento. 
Do total analisado, em 40,5\% dos idosos, foi constatado algum tipo de enteroparasitose, sendo que as helmintíases, com 76,4\%, foram predominantes. A Tabela 1 mostra os parasitas intestinais encontrados nessa pesquisa por ordem de prevalência.

TABELA 1 - Prevalência de enteroparasitas na população geronte de Parnaíba, Piauí.

\begin{tabular}{lc}
\hline Parasita & Porcentagem (\%) \\
\hline Entamoeba coli & 50,4 \\
Ascaris lumbricoides & 50,4 \\
Entamoeba histolytica/dispar & 19,3 \\
Giardia lamblia & 11,8 \\
Ancilostomídeos & 2,5 \\
Enterobius vermicularis & 1,7 \\
Strongyloides stercoralis & 0,8 \\
Trichuris trichiura & 0,8 \\
Schistosoma mansoni & 0,8 \\
\hline
\end{tabular}

A partir da análise dos questionários, 5,1\% dos idosos afirmaram não ter vaso sanitário em casa, realizando as necessidades fisiológicas em áreas próximas à residência. Quanto ao destino do lixo, alguns idosos admitiram queimar $(1,7 \%)$, enterrar $(2,4 \%)$ ou jogar o lixo domiciliar em terrenos baldios (34\%), por não haver coleta periódica na rua onde moram. No item proximidades à casa, observou-se a existência de lama e/ou água empoçada (22,1\%), lixo e/ou dejetos (15\%), entulho (3\%) e animais vadios (13,9\%). Cerca de $30,6 \%$ dos idosos admitiram que o piso de suas residências eram de barro, terra, cimentado ou piso morto. Em 77,9\% dos domicílios, havia presença de animais e em 49,7\% havia algum tipo de praga na residência.

O total de 1,4\% dos entrevistados utilizavam água advinda de poços manuais ou do rio para beber, lavar roupas e alimentos e $17 \%$ admitiram não fazer nenhum tipo de tratamento de água antes de beber. Cerca de $80,3 \%$ dos idosos afirmaram consumir frutas e verduras lavadas, sendo que $69,7 \%$ compram esses alimentos em mercados municipais. Aproximadamente, $61,6 \%$ responderam consumir carne oriunda desses pontos de venda.

O histórico clínico revelou que alguns idosos apresentavam sintomatologia para as enteroparasitoses, como diarreia $(20,4 \%)$, vômito $(11,2 \%)$, prurido anal $(18,7 \%)$ e dor abdominal $(21,8 \%)$.

Aproximadamente, $17 \%$ dos idosos nunca utilizaram qualquer tipo de medicação antiparasitária. Cerca de $19 \%$ tomou pela última vez esse tipo de medicação há mais de 5 anos. Em torno de $69,7 \%$ dos idosos, afirmaram que não se deslocam periodicamente para outras localidades e $26,5 \%$ afirmaram não usar calçados constantemente.

Os resultados desta pesquisa são semelhantes aos achados de Oliveira e cols ${ }^{7}$, realizados em Parnaíba. Isso sugere que após praticamente uma década as condições sanitárias e sociais da cidade continuam favorecendo a disseminação dessas doenças.

Neste trabalho, as helmintíases foram as enteroparasitoses predominantes, enquanto pesquisas realizadas no sudeste piauiense denotam prevalência de protozoários ${ }^{9}$. Isto pode ser explicado pelo fato de que o sudeste do Piauí faz parte de uma região semi-árida, que dificulta a proliferação de helmintos. Segundo Alves cols ${ }^{9}$, a disseminação das helmintíases na região nordeste do país está intimamente relacionada com a umidade do solo.

Em algumas amostras de fezes, foram detectados ovos de ancilostomídeos. Visto que parcela significativa dos idosos afirmou não utilizar calçados constantemente, é possível que esteja ocorrendo contaminação por mecanismos ativos cutâneos ${ }^{10}$.

Assim como os trabalhos de Silva e Silva ${ }^{10}$, a ocorrência de poucos casos de Enterobius vermicularis e nenhuma ocorrência de Taenia sp pode estar subestimada, devido à realização de métodos não-específicos para estes agentes.

As condições higiênico-sanitárias se mostraram estritamente relacionadas com altas prevalências de enteroparasitos em vários trabalhos realizados no Brasil ${ }^{7,9,10,11}$. Nesta pesquisa, não foi diferente. A falta de vasos sanitários nas residências, a presença de lama, água empoçada, dejetos, entulho e animais vadios, aliados a uma coleta de lixo ineficaz, mostraram que Parnaíba possui fatores que predispõem a disseminação de enteroparasitas. Tendo em vista essas precárias condições, sugere-se que alguns indivíduos tenham sido parasitados na própria região onde moram, uma vez que grande parte dos idosos afirmou não se deslocar periodicamente para outras localidades.

$\mathrm{O}$ fato de uma parcela da população não fazer nenhum tipo de tratamento de água antes de beber tornam-a alvo mais comum de doenças, como as enteroparasitoses ${ }^{6}$. Komagome cols ${ }^{11}$, correlacionando a prevalência de enteroparasitoses em crianças com seus aspectos epidemiológicos, revelaram que o tipo de água utilizada para consumo foi um fator de risco detectado, uma vez que quem consumiu água não-filtrada apresentou 15,9 vezes mais chances de adquirir enteroparasitoses.

Apesar da maioria dos idosos consumirem frutas e verduras lavadas, a origem da água utilizada não garante que estes alimentos estejam isentos de contaminação. $O$ grau de contato com os alimentos já corresponde a um fator de risco ${ }^{4}$. Trabalhos feitos pelo Grupo de Pesquisa em Parasitologia Biomédica da Universidade Federal do Piauí (UFPI), ainda não publicados, revelaram que $41,6 \%$ das alfaces analisadas provenientes de feiras-livres de Parnaíba apresentaram-se contaminadas por larvas de Strongyloides sp e cistos de protozoários.

O fato da maioria dos idosos consumirem carnes e derivados oriundos de mercados municipais pode representar um risco à saúde dessa classe. A maioria desses pontos de vendas da Cidade de Parnaíba apresenta condições deficitárias de higiene, facilitando a disseminação de doenças transmitidas por alimentos.

Os idosos habitualmente realizam atividades domésticas que exigem contato direto com o solo, como cultivo de hortas ${ }^{12}$. Trabalhos realizados no Rio de Janeiro mostraram que 30\% das amostras de solo analisadas estavam contaminadas por ovos de ancilostomídeos e Toxocara $\mathrm{sp}^{13}$. Aliado a estas tarefas caseiras, uma percentagem significativa dos idosos admitiu que o piso de suas residências era de barro, terra, cimentado ou piso morto. $\mathrm{O}$ contato com o solo úmido é um fator que facilita a transmissão de geohelmintos ${ }^{9}$, como Ascaris lumbricoides, observado em alta prevalência nessa pesquisa.

A maioria dosidosos afirmou possuir algum tipo de animal em casa. A convivência do ser humano com animais domésticos acarreta melhoria nas condições de vida dos idosos ${ }^{14}$. Todavia, muitos animais constituem uma importante fonte de infecção parasitária ${ }^{15}$. Sugere-se que os idosos parasitados por Giardia lamblia tenham adquirido esse agente dentro da própria casa, visto que essa patologia possui potencial zoonótico ${ }^{15}$ e que em praticamente na metade dos domicílios havia algum tipo de praga, sobretudo insetos, podendo servir como vetores de doenças.

Quando se correlaciona idosos e enteroparasitoses, observa-se que não existe uma sintomatologia específica para este grupo ${ }^{8}$. Os danos que os enteroparasitas podem causar incluem anemia, diarréia, 
obstrução intestinal e má absorção $0^{3,6,7,10}$. A manifestação clínica dessas patologias é variável e algumas vezes inespecífica. Geralmente, a sintomatologia é proporcional ao número de parasitos albergados ${ }^{6}$.

O fato de parte dos idosos nunca ter usado nenhum tipo de medicação antiparasitária, ou que tenha usado medicamento do gênero a mais de cinco anos, leva a crer que a alta prevalência de enteroparasitoses se deve também ao grau de descomprometimento do idoso com sua própria saúde. Todavia, isso não poder ser atribuído como fator principal para o resultado observado, uma vez que deve ser levada em consideração a questão das deficiências imunológicas observadas na terceira idade e a associação dos enteroparasitas com doenças advindas do envelhecimento ${ }^{3,4,8}$. Além disso, o difícil acesso ao sistema público de saúde traz consigo dificuldades para erradicação de doenças. É preciso ressaltar também a importância do profissional da saúde frente à orientação dessa classe no que diz respeito ao combate contra essas doenças ${ }^{6}$.

Conclui-se que existe alta prevalência de enteroparasitoses na população geronte de Parnaíba, indicando um estado epidemiológico preocupante. Deste modo, é nítida a necessidade de implantação de novas políticas públicas de saúde, voltadas tanto para a melhoria das condições sociais quanto da situação ambiental em que a população está inserida. Todavia, de nada adianta a intervenção com programas de controle se a população idosa não se conscientizar que é necessária a adoção de medidas de educação preventiva, uma vez que seus maus hábitos podem desempenhar papel decisivo na disseminação dessas patologias.

\section{AGRADECIMENTOS}

Os autores agradecem ao Campus Ministro Reis Velloso e ao grupo Rede FIBRA, em especial às pesquisadoras Priscila Mesquita, Amanda Campelo, Vanessa Soares, Vanessa Menezes, Luzeni Garcez e Edésio Borges.

\section{CONFLITO DE INTERESSE}

Os autores declaram não haver nenhum tipo de conflito de interesse no desenvolvimento do estudo.

\section{SUPORTE FINANCEIRO}

Programa Institucional de Bolsa de Iniciação Científica (PIBIC).

\section{REFERÊNCIAS}

1. Motta LB, Aguiar AC. Novas competências profissionais em saúde e o envelhecimento populacional brasileiro: integralidade, interdisciplinaridade e intersetorialidade. Cienc Saude Coletiva 2007; 12:363-372.

2. Veras R. Population aging today: demands, challenges and innovations. Rev Saude Publica 2009; 43:1-7.

3. Araujo CFF, Correia JS. Frequência de parasitas intestinais em idosos dos núcleos da Prefeitura de João Pessoa, Estado da Paraíba. Rev Bras Anal Clin 1997; 29:230-231

4. Ludwig KM, Frei F, Alvares Filho F, Ribeiro-Paes JT. Correlação entre condições de saneamento básico e parasitoses intestinais na população de Assis, Estado de São Paulo. Rev Soc Bras Med Trop 1999; 32:547-555.

5. Wu D, Meydani SN. Age-associated changes in immune and inflammatory responses: impact of vitamin E intervention. J Leukoc Biol 2008; 84:900-914.
6. Marques MC. Investigação parasitológica com ênfase nos Complexos E. histolytica/E. dispar e Teníase-Cirticercose em uma comunidade rural do sudeste do Piauí [dissertação]. [Niterói]: Universidade Federal Fluminense; 2003.

7. Oliveira MF, Costa STCB, Bezerra FSM. Incidência de enteroparasitos na zona rural do Município de Parnaíba, Piauí. Rev Bras Anal Clin 2001; 33:45-48.

8. Hurtado-Guerrero AF, Alencar FH, Hurtado-Guerrero JC. Ocorrência de enteroparasitas na população geronte de Nova Olinda do Norte - Amazonas, Brasil. Acta Amaz 2005; 35:487-490.

9. Alves JR, Macedo HW, Ramos Jr AN, Ferreira LF, Gonçalves MLC, Araújo A Parasitoses intestinais em região semi-árida do Nordeste do Brasil: resultados preliminares distintos das prevalências esperadas. Cad Saude Publica 2003; 19:667-670

10. Silva LP, Silva RMG. Ocorrência de enteroparasitos em centros de educação infantil no município de Patos de Minas, MG, Brasil. Biosci J 2010 , 26:147-151.

11. Komagome SH, Romagnoli MPM, Previdelli ITS, Falavigna DLM, Dias MLGG, Gomes ML. Fatores de risco para infecção parasitária intestinal em crianças e funcionários de creche. Cienc Cuid Saude 2007; 6:442-447.

12. Hurtado-Guerrero AF. Fatores determinantes do estado nutricional do idoso de Nova Olinda do Norte - Amazonas [dissertação]. [Manaus]: Instituto Nacional de Pesquisa da Amazônia, Universidade do Amazonas; 2000.

13. Brener B, Mattos DPBG, Millar PR, Arashiro EKN, Duque-Ferreira V, Sudré AP. Estudo da contaminação de praças públicas de três municípios do Estado do Rio de Janeiro, Brasil, por ovos e larvas de helmintos. Rev Patol Trop 2008; 37:247-254.

14. Asano K, Suzuki K, Matsumoto T, Sakai T, Asano R. Prevalence of dogs with intestinal parasites in Tochigi, Japan in 1979, 1991 and 2002. Vet Parasitol 2004; 120:243-248.

15. Capuano DM, Rocha GM. Ocorrência de parasitas com potencial zoonótico em fezes de cães coletadas em áreas públicas do município de Ribeirão Preto, SP, Brasil. Rev Bras Epidemiol 2006; 9:81-86. 